Upcoming: April 2-7, 2nd World Congress on Microwave Processing, Endorsed: April 24-28, MRS Spring Meeting, MRS.

\begin{abstract}
To list an event in the Calendar, contact J. Meiksin, Materials Research Society, 506 Keystone Drive, Warrendale, PA 150867573; 724-779-3004 ext. 522; fax 724-779-8313.

MRS, A-MRS, C-MRS, E-MRS, MRS-A, MRS-I, MRS-J, MRSK, MRS-R, MRS-T, M-MRS, or IUMRS at the end of an entry indicates sponsorship or co-sponsorship of an event by the International Union of Materials Research Societies or one of its adhering bodies. "Endorsed" identifies events endorsed by MRS.
\end{abstract}

$\boldsymbol{\nabla}$ identifies a new or revised entry this month.

See the January 2000 MRS BULLETIN for March 2000 Calendar entries.

\section{APRIL 2000}

2-5 $\nabla$ AWS Intl. Brazing and Soldering Conf., Albuquerque, NM. American Welding Society, 550 N.W. Leleune Rd., Miami, $\mathrm{F}$ 33126; 305-443-9353; fax 305443-7559; e-mail info@aws.org; www.aws.org.

\section{2-7 2nd World Congress on} Microwave Processing, Orlando, R. D.E Gark, Organizing Committee Chair, Dept. of Materials Science and Engineering 136 MAE, Univ. of Horida, P.O Box 116400, Gainesville, FL 32611-6400; 352-392-3660; fax 352-846-2033. Endorsed.

3-4 Microscopy of Composite Materials V, Oxford, UK Royal Microscopical Society, 37/38 St. Cements, Oxford OX4 1AJ, UK; 44-1865-248768; fax 44-1865791237; email meetings@rms. org.uk; http://www.rms.org.uk.

\section{3-4 $\boldsymbol{\nabla}$ POMI Conf. on PreEch} and Post-Ech Processes, San Diego, CA J. Grande, Executive Director, Photo Chemical

Machining Inst., P.O. Box 2403, Littleton, MA 01460; 978-4863746; fax 978-486-4915; e-mail photomach@aol.com.

3-5 $\nabla$ IMAPS and NASA Workshop on Eectronic Parts and Packaging for Space and Aeronautic Applications, Washington, $D C$. Intl. Microelectronics And Packaging Society And Educational Foundation, 1850 Centennial Park Dr., Ste. 105, Reston, VA 201911517; 703-758-1060; fax 703-7581066; email IMAPS@imaps.org.
3-6 3rd Intl. Conf. on Eectromagnetic Processing of Materials, Nagoya, Japan. JATS Sogo-Kojimachi, No. 3 Bldg., 1-6 Kojimachi, Chiyoda Ku, Tokyo 102-0083, Japan; 81-3-32394804; fax 81-3-3239-4714; e-mail jatisc@msn.com.

9-14 Nanostructured Sensors and Other Gas-Reactive Applications, Barga, Tuscany, Italy. United Engineering Foundation Conferences, 3 Park Ave., 27th Hoor, New York, NY 10016-5902 212-591-7836; fax 212-591-7441; e-mail engfnd@aol.com; http://www.engfnd.org.

10-11 SPIE Regional Meeting on Optoelectronics, Photonics, and Imaging, Albuquerque, NM. SPIE, P.O. Box 10, Bellingham, WA 98227-0010; 360-676-3290; fax 360-647-1445; e-mail

spie@spie.org; www.spie.org.

10-12 $\nabla$ 5th Intl. Conf. on Sted Mill Wastes and By-Products, Atlanta, GA C. Jones, Gorham Conferences, Inc., 211 Mosher Rd., Gorham, ME 04038; 207-8925445; fax 207-892-2210; e-mail gorham@goradv.com.

11-13 Intl. Microscopy Conf., London, UK Royal Microscopical Society, 37/38 St. Gements, Oxford OX4 1AJ, UK; 44-1865248768; fax 44-1865-791237; e-mail meetings@rms.org.uk; http://www.rms.org.uk.

12-14 Materials Congress 2000 and Materials Industries Exhibition, Wiltshire, UK H. Kaune, IOM, 1 Carlton House Terrace, London SW1Y5DB, UK; 44-20-7451-7360; fax 44-20-7451-7349; email hildakaune@materials.org.uk; uww.materials.org.uk.

12-16 $\nabla$ EURESCOConf. on Organic Eectrochemistry in the New Century: Advances in Sensing, Recycling, and Cean Conversions, Tomar, Portugal.

J. Hendekovic, European Science Foundation, 1 quai LezayMarnésia, 67080 Strasbourg Cedex, France; 33-388-767135; fax 33-388-366987; e-mail euresco@esf.org; www.esf.org/euresco.

15-20 43rd SVC Annual Conf. Denver, $\infty$. Society of Vacuum Coaters, 71 Pinon Hill Place, N.E, Albuquerque, NM 87122-1914 505-856-7188; fax 505-856-6716; email svcinfo@svc.org;

http://www.svc.org
16-19 6th Intl. Multidisciplinary Conf.: Complexity and Fractals in the Sciences, Singapore. http://www.kingston. ac.uk/fractal.

16-21 $\nabla$ Conf. on Molecular and Ionic Qusters, Toulouse, France.

P. Brechignac, Labo.

Photophysique Moleculaire, Univ. de Paris-Sud, Bat. 210, F91405

Orsay Cedex, France;

e-mail Philippe.Brechignac@ppm.

u-psud.fr; www.irsamc.ups-

tlse.fr/irsamc/mic2000.htm.

23-28 Conf. on High Power Laser Ablation, Santa Fe, NM. SPIE, P.O. Box 10, Bellingham, WA 98227-0010; 360-676-3290; fax 360-647-1445; e-mail spie@spie.org; www.spie.org.

24-28 MRS Spring Meeting San Francisco, CA. Materials Research Society, 506 Keystone Drive, Warrendale, PA 150867573; 724-779-3003; fax 724-779 8313; http://www.mrs.org/. MRS.

25-28 $\nabla$ AWS Intl. Welding and Fabricating Convention, Chicago, IL. American Welding Society, 550 N.W. Leleune Rd., Miami, $\mathrm{P}$ 33126; 305-443-9353; fax 305443-7559; e-mail info@aws.org; unw.aws.org.

25-29 22nd Annual Intl. High Technology, Safety, Industrial Hygiene, and Environmental Conf. Arlington, VA Semiconductor Safety Association, 1313 Dolley Madison Blvd., Ste. 402, McLean, VA 22101; 703-790-1745; fax 703790-2672; email ssa@burkinc.com; http://www. semiconductorsafety.org.

26-28 $\nabla$ 8th Intl. Conf. on Metrology and Properties of Engineering Surfaces, West Yorkshire, England. L. Rees; 44-1484-472312; fax 44-1484451883; email e.arees@hud.ac.uk; unw.hud.ac.uk/schools/engineering/ welcome.htm.

26-28 Intl. Conf. on High Density and Systems Packaging, Denver, $\infty$. Intl. Microelectronics and Packaging Society, 1850 Centennial Park Dr., Ste. 105, Reston, VA 20191-1517; 888-464-6277; fax 703-758-1066; e-mail imaps@imaps.org; www.imaps.org.

26-30 2nd Intl. Conf. on Atomic and Molecular Data and Their Applications, Oxford, UK K. Berrington; email k.berrington@ shu.ac.uk; http://physics.nist.gov/ icamdata.

29-2 APS April Meeting, Long Beach, CA. APS Headquarters, One Physics Elipse, College Park, MD 20740-3844; 301-209-3200; fax 301-209-0865; e-mail meetings@ aps.org; http://www.aps.org.
30-3 ACerS 102nd Annual Meeting, St. Louis, MO. Oustomer Service Dept., The American Ceramic Society, P.O. Box 6136, Westerville, OH 43086-6136; 614-794-5890; fax 614-899-6109; e-mail customersrvc@acers.org; www.acers.org.

\section{MAY 2000}

1-3 6th Intl. Conf. on Remote Sensing for Marine and Coastal Environments, Charleston, SC. QRIM/Marine Conf., P.O. Box 134008, Ann Arbor, Ml 48113-4008; 734-994-1200; fax 734-994-5123; http://www.erim-int.com/conf/ conf.html.

5-6 APS Ohio Section Spring Meeting, Oncinnati, OH. M. Ma, Univ. of Oncinnati; e-mail michael.ma@uc.edu; nww.aps.org.

7-12 Conf. on Lasers and Eectro-Optics, and Quantum Eectronics and Laser Science Conf., San Francisco, CA Optical Society of America, Conf. Services Dept., 2010 Massachusetts Ave. N.W., Washington, DC200361023; 202-416-1980; fax 202-4166100; e-mail confserv@osaorg; http://www.osa.org/mtg-conf.

13-18 $\nabla$ Molecules of Biological Interest in the Gas Phase:

Experimental Tools and Quantum Chemistry EuroConference, Les Houches, France. J. Hendekovic, European Science Foundation, 1 quai Lezay-Marnésia, 67080 Strasbourg Cedex, France; 33-388 767135; fax 33-388-366987; e-mail euresco@esf.org www.esf.org/euresco.

14-16 Conf. on Auoropolymers, Maui, HI. SPI Ruoropolymer Div., 1801 KSt. N.W., Ste. 600K, Washington, DC20006; 202-974-5234.

\section{4-17 Conf. on Optical Data}

Storage, Vancouver, BC, Canada SPIE, P.O. Box 10, Bellingham, WA 98227-0010; 360-676-3290; fax 360-647-1445; email spie@spie.org; www.spie.org

14-18 12th Intl. Conf. on Indium Phosphide and Related Materials, Williamsburg, VA. IPRM'00, IIIILEOS, 445 Hoes Lane, P.O. Box 1331, Piscataway, NN 08855-1331; 732-562-3898; fax 732-562-8434 http://www.iee.org/leos.

14-19 197th Meeting of The Eectrochemical Society, Toronto, Ontario, Canada The Bectrochemical Society, $65 \mathrm{~S}$. Main St., Pennington, NN 08534-

2839; 609-737-1902; fax 609-737-2743; e-mail ecs@electrochem.org; http://www.electrochem.org/ meetings/.

15-20 6th World Biomaterials Congress, Kamuela (Big Island), HI. Society for Biomaterials, 13355 10th Ave. N., Ste. 108 Minneapolis, MN 55441; 612-9278108; fax 612-927-8127; e-mail biomaterials.org; http://www. biomaterials.org. 
22-24 Conf. on Soft Magnetic Materials, Dearborn, MI. D. Crommett, Intertech Conferences, 19 Northbrook Dr., Portland, ME 04105; 207-781-9800; fax 207781-2150; e-mail deboraho@intertech usa.com.

22-26 5th Intl. Symposium on High-Temperature Corrosion and Protection of Materials, lle des Embiez, France. http://www.up. univ-mrs.fr/ cdd/ 5th-ishtc.html.

22-27 26th Congress on Chemical Engineering,

Environmental Protection, and Biotechnology, Frankfurt am Main, Germany. DECH日MA Deutsche Gesellschaft fur Chemisches Apparatewesen, Chemische Technik und Biotechnologie e.V., Th. Scheuring, Germany; 49-697564-230; fax 49-69-7564-298; http://www.dechema.de.

\section{2-28 7th Conf. on}

Intersections Between Particle and Nuclear Physics, Quebec Oity, Canada A.B. Macinnis; 617-253 9285; e-mail macinnis@mit.edu; http://CPANP.mid.edu.

24-26 3rd Intl. Conf. on Advances in Fuid Mechanics, Montreal, Canada Conf. Secretariat HPC2000, Wessex Inst. of Technology, Ashurst Lodge, Ashurst, Southampton, SO40 7AA, UK; 44-238-029-3223; fax 44-238-029-2853; email wit@wessex.ac.uk; http://www.wessex.ac.uk/conferences/2000.

28-2 10th Intl. Offshore and Polar Engineering Conf., Seattle, WA Intl. Society of Offshore and Polar Engineers, P.O. Box 1107, Golden, C080402-1107; 303-420-8114; fax 303-420-3760.

28-2 Intl. Conf. on Transport Processes in Inorganic Materials: Fundamentals to Devices, Venice, Italy. GMTEC, P.O. Box 174 48018 Faenza, Italy; 39-54622461; fax 39-546-664138.

29-2 Centrifugal Materials Processing IV-4th Intl. Workshop on Materials Processing at High Gavity, Potsdam, NY. L.L. Regel Carkson Univ., Potsdam, NY 13699-5814; 315-268-7672; fax 315-268-3833; e-mail regel@ clarkson.edu; http://www.clarkson. edu/regel/workshop.htm.

30-2 Intl. Conf. on Bectronic Materials and E-MRS Spring Meeting, Strasbourg, France. Materials Research Society, 506 Keystone Dr., Warrendale, PA 15086-7573; 724-779-3003; fax 724-779-8313; http://wwwemrs.c-strasbourg.fr. E-MRS, IUMRS.
30-3 Intl. Conf. on Powder Metallurgy and Particulate Materials, New York, NY. T. Stillman, Metal Powder Industries Federation, 105 College Rd. East, Princeton, NJ 08540-6692; 609452-7700; fax 609-987-8523; e-mail stillman@mpif.org; www.mpif.org

\section{JUNE 2000}

5-7 Extreme High Vacuum and Surface Conditioning, Newport News, VA H.F. Dylla, Jefferson Lab., 1200 Jefferson Ave., Newport News, VA23606; 757269-7450; fax 757-269-6357; e-mail dylla@jlab.org.

12-16 6th Intl. Conf. on Molten Slags, Huxes, and Salts, Stockholm, Sweden. Congrex Sweden AB, P.O. Box 5619, SE-114 86, Stockholm, Sweden; 46-8-459-6600; fax 46-8-6619125; e-mail slags2000@congrex.se; http://www.slags2000.kth.se.

12-16 Intl. Conf. on Applications of Photonic Technology, Quebec Oity, Canada SPIE, P.O. Box 10, Bellingham, WA 98227-0010; 360-676-3290; fax 360-647-1445; e-mail spie@spie.org; www.spie.org.

\section{4-17 31st Annual Meeting} of the APS Division of Atomic, Molecular, and Optical Physics, Storrs, CT. D. Dole, Ar. for Professional Development, Univ. of Connecticut, U-56D, One Bishop arcle, Storrs, CT 06269-4056; 860-486-1072; fax 860-486-5221; email ddole@access.ced.uconn.edu.

18-22 13th Topical Conf. on High-Temperature Plasma Diagnostics, Tuscon, AZ C.W. Barnes, P-24 MS-E26, Los Alamos National Lab., Los Alamos, NM 87545; 505-665-5687; fax 505-665-3552; e-mail cbarnes@anl.gov; www.lanl.gov/HTPD2000/

18-23 OSA Diffractive Optics and Micro-Optics Topical Meeting, collocated with OSA Optical

Fabrication and Testing Topical

Meeting, Quebec City, Canada.

Optical Society of America, Conf.

Services Dept., 2010

Massachusetts Ave., N.W.,

Washington, DC20036-1023;

202-416-1980; fax 202-416-6100; e-mail confserv@osaorg; http://www.osa.org/mtg-conf.

19-21 Intl. Conf. on Computational Methods for Smart Structures and Materials, Madrid, Spain. S. Hanley, Wessex Inst. of Technology, Ashurst Lodge, Ashurst, Southampton, SO40 7AA UK; 44-238-029-3223; fax 44-238029-2853; e-mail shanley@wessex. ac.uk; http://www.wessex.ac.uk/ conferences/2000/.
19-23 23rd Meeting of the Bectrochemistry Group of the Spanish Royal Society of Chemistry, San Sebastián, Spain. T. Fernández Otero, Lab. of Eectrochemistry, Univ. of the Basque Country, P.O. Box 1072 , 20080 San Sebastián, Spain; 34 943-018186; fax 34-943-212236; e-mail qppfeott@sq.ehu.es.

21-23 4th Intl. Meeting on Vector and Parallel Processing, Porto, Portugal. Viagens Abreu SA; e-mail congress.porto@abreu.pt; www.fe.up.pt/vecpar2000/

21-23 42nd Bectronic Materials Conf., Denver, 00 . Oustomer Service, TMS, 184 Thorn Hill Rd. Warrendale, PA 15086-7528; 724 776-9000; fax 724-776-3770; e-mail tmsgeneral@ms.org; http://www.tms.org/cms

21-23 $\nabla$ 60th Physical Bectronics Conf., Baton Rouge, LA. R.L. Kurtz, Dept. of Physics and Astronomy, 202 Nicholson Hall, Louisiana State Univ., Baton Rouge, LA 70803; 225-388-4028 fax 225-388-5855; www. physicalelectronics.org.

21-23 Intl. Conf. on Mobile and Rapidly Assembled Structures, Madrid, Spain. S. Hanley, Wessex Inst. of Technology, Ashurst Lodge, Ashurst, Southampton, SO40 7AA, UK; 44-238-029-3223; fax 44-238-029-2853; e-mail shanley@wessex.ac.uk; http://www.wessex.ac.uk/ conferences/2000/.

24-29 $\nabla$ EURESOOConf. on Guster-Surface Interactions: Applications, Castelvecchio Pascoli, Italy. J. Hendekovic, European Science Foundation, 1 quai Lezay-Marnésia, 67080

Strasbourg Cedex, France; 33-388-767135; fax 33-388366987; e-mail euresco@esf.org; www.esf.org/euresco.

26-28 6th Intl. Conf. on Advanced Computational Methods in Heat Transfer, Madrid, Spain. Conf. Secretariat Heat Transfer 2000, Wessex Inst. of Technology, Ashurst Lodge, Ashurst,

Southampton, SO40 7AA, UK; 44 238-029-3223; fax 44-238-029 2853; e-mail wit@wessex.ac.uk; http://www.wessex.ac.uk/ conferences/2000.

\section{6-2 Seminar on Defect} Structures Evolution in Condensed Matter, Barnaul, Russia 日DS 2000, General Physics Dept., Altai State Technical Univ., 46 Lenin St. Barnaul, Russia 656099; 007 3852-368-0522; fax 007-3852260-516; e-mail asba@ab.ru (mailto:asba@ab.ru); http://www. dcn-asu.ru/structure/cdo/ index.ru.html.
28-30 Merton C. Femings Symposium, Cambridge, MA Oustomer Service, TMS, 184 Thorn Hill Rd., Warrendale, PA 15086-7528; 724-776-9000; fax 724-776-3770; e-mail tmsgenera @tms.org; http://www.tms.org/cms

\section{JULY 2000}

1-6 $\nabla$ EuroConference on the Physics and Applications of MultiJunctions Superconducting Josephson Devices, Acquafredda di Maratea, Italy. J. Hendekovic, European Science Foundation, 1 quai Lezay-Marnésia, 67080 Strasbourg Cedex, France; 33-388767135; fax 33-388-366987; e-mail euresco@esf.org; www.esf.org/euresco.

2-7 $\nabla$ Gordon Research Conf. on Tribology, Plymouth, NH. D.A

Rigney, Ohio State Univ., 2041 College Rd., Columbus, $\mathrm{OH} 43210$ 614-292-1775; fax 614-292-1537; e-mail rigney.1@osu.edu; www.grc.uri.edu/programs/ 2000/tribo.htm

3-7 11th Intl. Conf. on Semiconducting and Insulating Materials, Canberra, Australia

C. Jagadish, Australian Natl. Univ. Research School of Physical Sciences and Engineering, Canberra, ACT 0200, Australia; 612-6249-0363; fax 61-2-6249-0511; e-mail simc2000@anu.edu.au; http://rsphysse.anu.edu.au/admin simc2000/.

3-7 $\nabla$ 26th Annual Intl. Conf. on Organic Coatings: Waterborne,

High Solid, and Powder Coatings, Athens, Greece. Inst. of Materials Science, Div. of Program

Organization, P.O. Box 369, New Paltz, NY 12561; 914-255-0757; fax 914-255-0978; e-mail icinquiry@ms-np.org; www.imsnp.org/.

9-14 12th European Congress on Bectron Microscopy, Brno, Czech Republic. http:// www.eurem2000.isibrno.cz.

10-12 6th Intl. Conf. on Residual Stresses, Oxford, UK. P. Farrelly, loM Conferences and Events; 44-171-451-7391; fax 44-171-839-2289; e-mail pauline farrelly@materials.org.uk.

10-12 OSA Optical Amplifiers and Their Applications Topical Meeting, Quebec City, Canada Optical Society of America, Conf. Services Dept., 2010

Massachusetts Ave., N.W., Washington, DC20036-1023 202-416-1980; fax 202-416-6100; e-mail confserv@osa.org; http://www.osa.org/mtg-conf

10-13 The Plutonium FuturesThe Science Conf., Santa Fe, NM. A. Liesse, Plutonium Futures-The 
26-28 $\nabla$ Conf. on Bectronic Structure and Magnetism in Complex Materials, Washington, DC. Organizing Committee, ESCM2000, Ar. for Computational Materials Science, Code 6391, Naval Research Lab., Washington, DC20375; 202-404-8627; fax 202 404-7546; http://cst www.nrl.navy. $\mathrm{mil} / \mathrm{escm} 2000$.

29-2 $\nabla$ AAPT Summer Meeting, Ontario, Canada AAPT Meetings Dept., One Physics Elipse, College Park, MD 20740; e-mail aapt_meet @aapt.org; www.aapt.org.

30-4 SPIEAnnual Meeting, San Diego, CA SPIE, P.O. Box 10, Bellingham, WA 98227-0010; 360-676-3290; fax 360-647-1445; e-mail spie@spie.org; http://www. spie.org.

31-4 Denver X-ray Conf., Denver, $\infty$. D. Haherty, Conf. Coordinator, ICDD, 12 Campus Blvd., Newtown Square, PA 190733273; 610-325-9814; fax 610-325 9823; e-mail flaherty@icdd.com; www.dxcicdd.com.

\section{AUGUST 2000}

6-11 7th International Symposium on Polymer Eectrolytes (ISPE7), Noosa, Queensland, Australia Astrid Nordmann, ISPE7 Conference Secretariat, Centre for Advanced Materials Technology, Monash University, Wellington Road, Cayton, Victoria, 3168, Australia 61-3-9905-5791; fax 61-3-99044998; email ispe7@eng.monash. edu.au; http://www.chem.monash. edu.au/electrolytes/ispe7/.

6-11 16th IUPAC Conf. on Chemical Thermodynamics, concurrent with 55th Calorimetry Conf. and 10th Symposium on Thermodynamics of Nuclear Materials, Halifax, Nova Scotia, Canada M.A. White, Dept. of Chemistry, Dalhousie Univ., Halifax, Nova Scotia B3H 4J3, Canada; phone and fax 902-4943894; email icct@is.dal.ca; http://is.dal.ca/_ICCT.

6-11 Intl. Conf. on Magnetism, Recife, Brazil. S. Rezende; 55-81 281-8450; fax 55-81-271-0359; e-mail smr@df.ufpe.br.

7-10 8th Asia Pacific Physics Conf., Taipei, Taiwan. O. Huang, Conf. Secretariat, c/o Inst. of Physics, Academia Sinica, Nankang 115, Taipei, Taiwan; 8862-27899602; fax 886-2-27829636; e-mail appc2k@phys.sinicaedu.tw.
13-16 Nonlinear Optics Topical Meeting, Maui, HI. Optical Society of America, Conf. Services Dept. 2010 Massachusetts Ave., N.W., Washington, DC20036-1023; 202-416-1980; fax 202-416-6100; e-mail confserv@osa.org; http://www.osa.org/mtg-conf.

13-18 $\nabla$ 12th American Conf. on Orystal Growth and Epitaxy, Vail, C. T. Gentile, AOOGE-12

Secretariat, P.O. Box 3233 Thousand Caks, CA 91359-0233; 805-492-7047; fax 805-492-4062; e-mail aacg@lafn.org uww.aml.arizona edu/aacg.

15-19 Conf. on Nuclear Structure, East Lansing, MI. S. Conroy; 517-333-6333; e-mail NS2000@nscl.msu.edu; www.nscl.msu.edu/conferences/ NS2000.

\section{6-20 12th Intl Conf. on} Textures of Materials, Montreal, Canada N. Procyshyn, Dept. of Mining and Metallurgical Engineering, McGill Univ., 3450 University St., Montreal, Quebec H3A 2A7, Canada; 514-398-4383, fax 514-398-7099; e-mail

norma@minmet.lan.mgill.ca; http://www.icotom12.ica.ca.

16-22 Jahn Teller Symposium, Boston, MA M. Kaplan, Simmons College and Boston Univ.; 617521-2727; e-mail kaplan@buphy. bu.edu.

20-23 39th Annual Conf. of Metallurgists, Otawa, Canada $\mathrm{H}$ Mostaghaci, Advanced Materials Group, Industry Canada, 235 Queen St., Otawa, ON K1A OH5, Canada; 613-954-5012; fax 613-952-4209; e-mail mostaghaci.hamid@ic.gc.ca

20-25 17th Intl Conf. on Raman Spectroscopy, Beijing, China $\mathrm{H}$. Zhou, Secretary General, Dept. of Physics, Peking Univ., Beijing 100871, China; 8610-6275-1750; fax 8610-6275-9117; e-mail icors@pku.edu.cn; http://uww.hkics.com/icors2000 or http://www.pku.edu.cn/others/ icors/icors.htm.

21-25 $\nabla$ 13th Intl. Symposium on Exoemission and Related Relaxation Phenomena, Latvia A. Katashev, 田MT Inst., Riga Technical Univ., 1. Kalku St., Riga LV-1658, Latvia; phone/fax 3717089383; e-mail katashev@latnet.Iv.

22-26 Ab initio (from Bectronic Structure) Calculations of Complex Processes in Materials, Schwaebisch Gmuend, Germany. e-mail psik-coord@daresbury.

ac.uk
26-30 15th European Conf. on Atomic and Molecular Physics of Ionized Gases, Miskolc-Lillafured, Hungary. Z Donko, c/o Eotvos Lorand Physical Society, $\mathrm{H}-1371$ Budapest, P.O. Box 433, Hungary; e-mail escampig@elft.mteszhu; http://elft.mtesz.hu/escampig2000.

26-31 Scientific Basis for Nuclear Waste Management Symposium. Sydney, Austrailia. Conference Secretariat, P.O. Box Q894, QVB Post Office, Sydney NSW 1230 Australia; 61 2-92624211; fax 6s1 2-9262-4265; e-mail facets@facetmanagement.com.au; https://www.amink.com.au/secure/ ansto2000.htm. MRS

27-30 Intl. Conf. on Spectroscopy of Ultra-Thin Fims and Related Systems, X'an, P.R China Y. Fang, Spectroscopy Lab. Experimental Cr., Capital Normal Univ., Beijing 100037, P.R. China; e-mail yfang@bnu.edu.cn; fax 8610-6841-6837.

\section{1-5 $\nabla$ EURESOOConf. on}

Supramolecular Chemistry: Reactivity and Catalysis in Supramolecular Systems, Urbino, Italy. J. Hendekovic, European Science Foundation, 1 quai Lezay Marnésia, 67080 Strasbourg Cedex, France; 33-388-767135; fax 33-388-366987; e-mail euresco@esf. org; www.esf.org/euresco.

\section{SEPTEMBER 2000}

1-6 $\nabla$ 日URESOOConf. on Reactivity in Organized Microstructures: Formation, Microstructure and Reactivity of Liquid Colloidal Systems, Mont Ste Odile, France. J. Hendekovic, European Science Foundation, 1 quai LezayMarnésia, 67080 Strasbourg Cedex, France; 33-388-767135; fax 33-388-366987; e-mail euresco@esf.org www.esf.org/euresco.

\section{2-7 $\boldsymbol{\nabla}$ 日URESCOConf. on} Inorganic Chemistry: Design and Properties of Low Nuclearity Meta Complexes, Achievements and Challenges of Organometallic Complexes in Homogeneous Catalysis, San Feliu de Guixols, Spain. J. Hendekovic, European Science Foundation, 1 quai LezayMarnésia, 67080 Strasbourg Cedex, France; 33-388-767135; fax 33-388-366987 e-mail euresco@esf.org; www.esf.org/euresco.

3-7 $\nabla$ Polycrystalline Semiconductors Conf., St. Malo, France. O. Bonnaud, GMV Univ. of Rennes I, 35042 Rennes Cedex, France; 33-299-286071; fax 33-299-281674; e-mail polyse 2000@univ-rennes1.fr.
3-8 Congress of Histochemistry and Oytochemistry, York, UK Royal Microscopica Society, 37/38 St. Cements Oxford OX4 1AJ, UK; 44-1865248768; fax 44-1865-791237; e-mail meetings@rms.org.uk; http://www.rms.org.uk.

4-9 12th Intl. Conf. on Ion Beam Modification of Materials, Porto Alegre, Brazil. IBMM 2000, Inst. de Fisica, URRGS, Campus do Vale, Caixa Postal 15051; 91501-970 Porto Alegre/RS, Brazil; 55-51-3166323; fax 55-51-316-6510; e-mail IBMM2000@if.ufrgs.br.

4-10 $\nabla$ 6th Seminar on Computational Physics, Granada Spain. J. Marro, Inst. "Carlos I" for Theoretical and Computational Physics, Facultad de Oencias, Univ. of Granada, 18071-Granada Spain; fax 34-958-242-862; e-mai cphys@ugr.es; http://ergodic.ugr. es/cp/.

6-9 $\nabla$ ACerS Pacific Coast Regional Meeting with Basic Science Div., San Francisco, CA American Ceramic Society, P.O Box 6136, Westerville, OH 430866136; 614-794-5890; fax 614-899 6109; e-mail customersrvc@ acers.org; www.ceramics.org

9-14 $\nabla$ EuroConference on Atoms and Molecules in SuperIntense Laser Felds, Acquafredda di Maratea, Italy. J. Hendekovic, European Science Foundation, 1 quai Lezay-Marnésia, 67080 Strasbourg Cedex, France; 33-388767135; fax 33-388-366987; e-mail euresco@esf.org; www.esf.org/euresco.

9-14 $\nabla$ Particle Physics and Gravitation: EuroConference on Quantum Fields and Strings, Crete, Greece. J. Hendekovic, European Science Foundation, 1 quai LezayMarnésia, 67080 Strasbourg Cedex, France; 33-388-767135; fax 33-388-366987; e-mail euresco @esf.org; www.esf.org/euresco.

9-15 Intl. Symposium on the Process Metallurgy of Uranium, Saskatoon, Canada C. Edwards, 306-956-6323; fax 306-956-6533; e-mail chuck_edwards@cameco. com.

10-14 ACerS Functionally Graded Materials Conf., Estes Park CO. Oustomer Service Dept., The American Ceramic Society, P.O.

Box 6136, Westerville, OH 430866136; 614-794-5890; fax 614-899 6109; e-mail customersrvc@acers. org; www.acers.org.

10-15 Conf. on Lasers and Bectro-Optics/Europe, and Intl. Quantum Bectronics Conf., Nice, France. Optical Society of America Conf. Services Dept., 2010

Massachusetts Ave., N.W. Washington, DC20036-1023; 202-416-1980; fax 202-416-6100 e-mail confserv@osa.org

http://www.osa.org/mtg-conf.

13-15 7th Intl. Conf. on Computer Methods in Composite Materials and Structures, Bologna Italy. Conf. Secretariat CADOOMP 
17-22 $\nabla$ Applied

Superconductivity Conf., Virginia

Beach, VA. Courtesy Associates,

2000 L St. NW, Ste. 710,

Washington, DC20036; 202-973-

8692; fax 202-331-0111;

e-mail asc@courtesyassoc.com.

18-22 $\nabla$ IMAPS 33rd Intl.

Symposium on Microelectronics,

Boston, MA Intl. Microelectronics And Packaging Society And

Educational Foundation, 1850

Centennial Park Dr., Ste. 105,

Reston, VA20191-1517;

703-758-1060; fax 703-758-1066;

e-mail IMAPS@imaps.org.

18-22 $\nabla$ Intl. Conf. on Materials and Coatings for Extreme

Environments Performance,

Orimea, Ukraine. Organizing

Committe "MEI2000," Inst. for

Problems of Materials Science,

3 Krzhyzhanovsky Str., 03142 Kiev,

Ukraine; 380-44-444-3231;

fax 380-44-444-2131;

e-mail chern@materials.kiev.ua

21-22 $\nabla$ Intl. Activated Carbon Conf., Pittsburgh, PA. B. Sherman, Professional Analytical and Consulting Services, 409 Meade Dr., Coraopolis, PA 15108; 724457-6576; fax 724-457-1214; e-mail hnpacs@aol.com; members. aol.com/hnpacs/pacs.htm.

24-27 ACerSManufacturing Workshop, Louisville, KY. Oustomer Service Dept., The American Ceramic Society, P.O. Box 6136, Westerville, OH 430866136; 614-794-5890; fax 614-899 6109; e-mail customersrvc@ acers.org; www.acers.org

25-27 Intl. Conf. on Tungsten and Refractory Metals, Annapolis, MD. Metal Powder Industries Federation; 105 College Rd. East, Princeton, NJ 08540-6692; 609452-7700; fax 609-987-8523; e-mail info@mpif.org;

www.mpif.org.

27-30 $\nabla$ Worlds in Transition: Technoscience, Gitizenship, and Oulture in the 21st Century; Conf. of the Society for Social Studies of Science and the European Assoc. for the Study of Science and Technology, Vienna, Austria U. Felt., Prof. of Social Studies of Science, Dept. of Philosophy of Science and Social Studies of Science, Univ. of Vienna, Sensengasse 8/10, A-1090 Vienna Austria; 43-1-4277-47611; fax 43-1-4277-9476; e-mail 4SEASST@univie.ac.at; wnw. univie.ac.at/wissenschaftstheorie
30-5 $\nabla$ 日URESOOConf. on Surface Engineering for Protection of Metals and Alloys, Acquafredda di Maratea, Italy. J. Hendekovic European Science Foundation, 1 quai Lezay-Marnésia, 67080 Strasbourg Cedex, France; 33-388767135; fax 33-388-366987; e-mail euresco@esf.org; unw.esf.org/euresco.

\section{OCTOBER 2000}

1-4 Brimacombe Memorial Symposium, Vancouver, Canada I. Samarasekera, Advanced Materials and Processing Engineering Lab. Univ. of British Columbia Rm. 111-2355 East Mall, Vancouver, BC, Canada V6T 1Z4; 604-8223668; fax 604-822-2965; e-mail indira@cmpe.ubc.ca.

\section{1-4 ACerS Glass and Optica} Materials Division Fall Meeting, Corning, NY. Oustomer Service Dept., The American Ceramic Society, P.O. Box 6136 Westerville, OH 43086-6136; 614-794-5890; fax 614-899-6109; e-mail customersrvc@acers.org; www.acers.org

\section{2-6 AVS 47th Intl.} Symposium, Boston, MA American Vacuum Society, 120 Wall St., 32nd Hoor, New York, NY 10005-3993; 212-248-0200; fax 212-248-0245; e-mail avsnyc@vacuum.org; http://www.vacuum.org

3-5 Advanced Metallization Conference (AMC) 2000, San Diego, California 510-642-4151:

fax 510-642-6027;

course@unx.berkeley.edu.

4-7 Meeting of the APS Division of Nuclear Physics, Williamsburg, VA. American Physical Society, One Physics Elipse, College Park MD 20740-3844; 301-209-3200; fax 301-209-0865; www.aps.org

\section{7-12 $\nabla$ EURESOOConf. on} Fundamental Aspects of Epitaxial Growth, Castelvecchio Pascoli, Italy. J. Hendekovic, European Science Foundation, 1 quai LezayMarnésia, 67080 Strasbourg Cedex, France; 33-388-767135; fax 33-388-366987;

e-mail euresco@esf.org; www.esf.org/euresco.

8-11 ACerSEectronics Division Fall Meeting, Cemson, SC. Oustomer Service Dept., The American Ceramic Society, P.O Box 6136, Westerville, $\mathrm{OH} 43086$ 6136; 614-794-5890; fax 614-8996109; e-mail customersrvc@acers. org; www.acers.org.

8-12 TMS Fall Meeting, St. Louis, MO. TMS, 184 Thorn Hill Rd., Warrendale, PA 15086; 724 776-9000; fax 724-776-3770; e-mail csc@tms.org; http://www. tms.org
11-13 14th Intl. Conf. on Optical Fber Sensors, Venice, Italy. A Grazia Mignani, IROE-ONR, Optics and Photonics Dept., Via Panciatichi 64, I-50127 Frenze Italy; 39-55-4235-262 fax 39-55-4379-569; e-mail mignani@iroe.fi.cnr.it.

14-16 13th Intl. Symposium on Superconductivity, Tokyo, Japan. M. Yoshida, ISS 2000 Secretariat, ISTEC, Shinbashi 5-34-3, Minatoku, Tokyo 105-0004, Japan; fax 81-3-3431-4044; e-mail josch da@istec.or.jp,www.istec.or.jp.

14-19 $\nabla$ EuroConference on Quantum Optics, Mallorca, Spain. J. Hendekovic, European Science Foundation, 1 quai LezayMarnésia, 67080 Strasbourg Cedex, France; 33-388-767135; fax 33-388-366987; e-mail euresco@esf.org; www.esf.org/euresco.

19-24 $\nabla$ High Performance Fibers: EuroConference on Fracture of Fibers, Mallorca, Spain. J. Hendekovic, European Science Foundation, 1 quai LezayMarnésia, 67080 Strasbourg Cedex, France; 33-388-767135 fax 33-388-366987; e-mail euresco@esf.org; www.esf.org/euresco.

20-21 $\nabla$ 2nd Intl. Symposium on Designing, Processing, and Properties of Advanced

Engineering Materials, Guilin, P.R China R. Yang, ISAEM Secretariat, Inst. of Metal Research, 72 Wenhua Rd., Shenyang 110015 , P.R. China; 86-24-2384-353155781; fax 86-24-2389-1320; e-mail ryang@pub.sy.In.cn; www.imr.ac.cn/ISABM-2000.htm/.

20-28 OSA Annual Meeting Providence, RI. Optical Society of America, Conf. Services Dept., 2010 Massachusetts Ave., N.W., Washington, DC20036-1023; 202-416-1980; fax 202-416-6100; e-mail confserv@osa.org; http://www.osa.org/mtg-conf.

22-25 TMS Fall Extraction and Process Metallurgy Meeting, Pittsburgh, PA. Oustomer Service TMS, 184 Thorn Hill Rd.

Warrendale, PA 15086-7528; 724-776-9000; fax 724-776-3770; e-mail csc@ims.org; http://www.tms.org/cms

22-27 198th Meeting of The Bectrochemical Society, Phoenix, $A Z$ The Bectrochemical Society, 65 S. Main St., Pennington, NJ 08534-2839; 609-737-1902; fax 609-737-2743; e-mail ecs@ electrochem.org; http://www. electrochem.org/meetings/.
23-27 Meeting of the APS Division of Plasma Physics, Quebec, Canada American Physical Society, One Physics 日lipse, College Park, MD 20740 3844; 301-209-3200; fax 301-2090865; www.aps.org

\section{9-3 $\nabla$ MCS/ACerS}

Manufacturing Workshop, Monterrey, Mexico. American Ceramic Society, P.O. Box 6136, Westerville, OH 43086-6136; 614 794-5890; fax 614-899-6109; e-mail customersrvc@acers. org; www.ceramics.org.

30-2 4th Intl. Conf. on High-Strength Low-Alloy Steels, X'an, China HSLA Steels 2000 Secretariat, CSM, 46 Dongsix Dajie, Beijing, China 100711; 86-10-6513-3925; fax 86-10-6512 4122; e-mail csm@public.bta net.cn

\section{NOVEMBER 2000}

1-4 16th Intl. Conf. on the Application of Accelerators in Research and Industry, Denton, TX J. Duggan; 940-565-3252; e-mail stippec@unt.edu; wuw.phys.unt.edu/accelcon/

2-4 Meeting of the APS Southeastern Section, Starkville MS. American Physical Society, One Physics Elipse, College Park MD 20740-3844; 301-209-3200; fax 301-209-0865; www.aps.org.

5-8 2nd Intl. Conf. on Processing Materials for Properties, San Francisco, CA. Oustomer Service, TMS, 184 Thorn Hill Rd., Warrendale, PA 15086-7528; 724-776-9000; fax 724-776-3770; e-mail csc@ tms.org; http://www.tms. org/cms

5-8 $\boldsymbol{\nabla}$ 6th Biennial Conf. on Organic Coatings, Hilton Head Island, SC. Inst. of Materials

Science, Div. of Program Organization, P.O. Box 369, New Paltz, NY 12561; 914-255-0757; fax 914-255-0978; e-mail narc@ ims-np.org; www.ims-np.org/.

6-8 $\nabla$ 14th Intl. Conf. and Workshops on Applied Geologic Remote Sensing, Las Vegas, $N$. Veridian-ERIM Intl., P.O. Box

134008, Ann Arbor, Ml 481134008; 734-994-1200;

fax 734-994-5123; www.erim-int. com/conf/conf.html.

8-10 $\nabla$ 3rd Biennial Conf. on Polymer Stabilizers and Modifiers, Hilton Head Island, SC. Inst. of Materials Science, Div. of Program Organization, P.O. Box 369, New Paltz, NY 12561; 914-255-0757; fax 914-255-0978; e-mail narc@ims-np.org; www.imsnp.org/.

12-17 AlChEAnnual Meeting Los Angeles, CA. AlChE 3 Park Ave., New York, NY 10016; http://www.aiche.org.

13-14 ASTM Symposium on Marine Corrosion in Tropical 


\section{Positions Available}

\section{FEBRUARY 2001 \\ 5-7 IEIEIntl. Solid-State} Grcuits Conf., San Francisco, CA D. Suiters, 202-331-2000; e-mail isscc@courtesyassoc.com.

\section{5-9 Advanced Research} Workshop on Semiconductor Nanostructures, Blenheim, New Zealand. D.J. Lockwood, Inst. fo Microstructural Sciences, Natl. Research Council of Canada, 1191 Montreal Rd., Bldg. M-36, Ottawa Ontario, Canada K1A 0R6; 613993-9614; fax 613-993-6486; e-mail david.lockwood@nrc.ca.

11-15 TMS Annual Meeting New Orleans, LA Oustomer Service, TMS, 184 Thorn Hill Rd. Warrendale, PA 15086-7528; 724 776-9000; fax 724-776-3770; e-mail tmsgeneral@tms.org; http://www.tms.org/cms.

\section{MARCH 2001}

12-16 APS March Meeting, Seattle, WA. American Physical Society, One Physics Elipse, college Park, MD 20740-3844; 301-209-3200; fax 301-209-0865; www.aps.org.

\section{5-30 199th Meeting of the} Bectrochemical Society, Washington, DC. The Bectrochemical Society, $10 \mathrm{~S}$. Main St., Pennington, NJ 085342896; 609-737-1902; fax 609-737 2743; e-mail ecs@electrochem.org; http://www.electrochem.org meetings/.

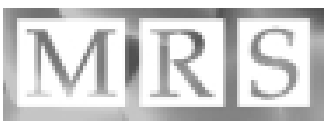

\section{APRIL 2001}

16-20 MRS Spring Meeting San Francisco, CA Materials Reserach Society, 506 Keystone Dr., Warrendale, PA 15086-7573; 724-779-3003; fax 724-779-8313; http://www.mrs.org. MRS

23-27 APS April Meeting Washington, DC. American Physical Society, One Physics Elipse, College Park, MD 20740 3844; 301-209-3200; fax 301-2090865; www.aps.org

\section{MAY 2001}

23-25 $\nabla$ 4th Intl. Conf. on Marine Technology, Szczecin, Poland. K. Savage, Wessex Inst. of Technology, WIT Marketing Coordinator, Ashurst Lodge Ashurst, Southampton SO40 7AA UK; 44-238-029-3223; fax 44-238-029-2853;

e-mail ksavage@wessex.ac.uk.

\section{JUNE 2001}

10-13 $\nabla$ 75th Colloid and Surface Science Symposium Pittsburgh, PA. S. Garoff, 412 268-6877; fax 412-681-0648; e-mail sg2e@andrew.cmu.edu.

17-20 $\nabla$ 5th Intl. Specia Emphasis Symposium on Superalloys $718,625,706$, and Derivatives, Pittsburgh, PA TMS, 184 Thorn Hill Rd., Warrendale, PA 15086; 724-776-9000; fax 724776-3770; e-mail

tmsgeneral@ms.org; www.tms.org/cms.

\section{OCTOBER 2001}

29-2 $\nabla$ Meeting of the APS Div. of Plasma Physics, Long Beach, CA APS Headquarters, One Physics Elipse, College Park MD 20740-3844; 301-209-3200; fax 301-209-0865; e-mail meetings@ aps.org; www.aps.org. MRS

\section{X-RAY MICRODIFFRACTION POSTDOCTORAL RESEARCH ASSOCIATE Materials Science \& Engineering Department University of Illinois at Urbana-C hampaign}

The Materials Science \& Engineering Department at the University of Illinois at Urbana-Champaign (UIUC) invites applications for a postdoctoral research associate to conduct $\mathrm{x}$-ray microbeam diffraction experiments at the Advanced Photon Source (APS), Argonne National Laboratory. Candidates must have an earned PhD degree in science or engineering, and must be within five years from the degree date. This is an opportunity for a motivated individual to contribute to the development of an important emerging area of materials research with access to unique experimental instrumentation. Knowledge of $\mathrm{x}$-ray materials characterization and synchrotron radiation techniques is desirable. Expertise with computer programming, electronic interfacing, and a willingness to develop new, interactive data collection and analysis approaches is essential.

The candidate will work closely with a team of scientists from UIUC and from the Oak Ridge National Laboratory (ORNL) to investigate the structure and evolution of materials on mesoscopic length scales using $x$-ray microbeam diffraction and fluorescence. The candidate will coordinate experiments with faculty at UIUC and will collaborate closely with scientists at UIUC, APS, and ORNL. The initial appointment will be for one year with the possibility of renewal. The opening is immediate with the application deadline of April 1, 2000, or until the position is filled. A resume and three letters of recommendation should be sent to Mr. J ay Menacher, Department of Materials Science and Engineering, University of Illinois, 1304 West Green Street, Urbana, IL 61801; Telephone: 217-244-6422; Fax: 217-333-2736; E-mail: jmenache@uiuc.edu. The University of Illinois is an Affirmative Action, Equal Opportunity Employer.

\section{PRINCIPAL SCIENTISTS \& RESEARCH ENGINEERS Nova Crystals, Inc.}

\section{COMPANY:}

Nova Crystals, Inc. (www.NovaCrystals. com) is a fabless photonics innovation house. The company is developing high-performance photonic devices for the optical networking, wireless communications, solid state lighting, and intra-chip communications markets. The company is located in San J ose, California.

\section{PRINCIPAL SCIENTIST:}

Nova seeks entrepreneurial-minded scientists or engineers to join Nova Crystals as Principal Scientists (PS) to help develop the company's materials and photonic device technologies. Reporting to the company's Vice President of R\&D, the PS will be responsible for innovative idea leadership and execution in the company's focus areas. The PS will also lead a team of PhDs in designing and prototyping devices based on the company's intellectual properties. The PS should be self-motivated with strong teamwork, leadership, and communication skills.

\section{RESEARCH ENGINEER:}

Nova seeks entrepreneurial-minded Research Engineers (RE) to help develop the company's materials and photonic device technologies. Reporting to the company's Principal Scientists, the RE will be responsible for "out-of-the-box" problem solving in the company's focus areas; and leading teams of internal and external partners in the design and prototyping of devices based on the company's intellectual properties. The RE should be self-motivated with strong teamwork, leadership, and communication skills.

\section{TECHNICAL REQUIREMENTS:}

The successful PS or RE candidate will have a PhD or equivalent degree in electrical engineering/applied physics/materials science and engineering with experience conducting research in an industrial or government lab. PS candidates should have $10+$ years' experience; RE candidates should have 3+years' experience. The successful candidate will have broad knowledge and deep expertise in III-V electronic and opto-electronic device physics and materials science. Typical devices including VCSELS (semiconductor and non-semiconductor based), visible LEDs, infrared LEDs, Avalanche detectors, and "smart-" III-V and Si wafers.

\section{COMPENSATION:}

The company offers a competitive compensation package that includes a base salary, an equity signing bonus, year-end performance-based bonuses in cash and equity, a competitive $401(\mathrm{~K})$ plan, and a comprehensive medical and dental plan. Applications will be accepted on a rolling basis. Interested candidates should send cover letter and CV to:

Kathie Anderson

Nova Crystals, Inc., 174 Component Drive, San J ose, CA 95131

E-mail: kathie@novacrystals.com 